\title{
Twelve Guidelines for Biological Sampling in Environmental Licensing Studies
}

\author{
Gonçalo Ferraz ${ }^{1,2 \star}$
}

${ }^{1}$ Biological Dynamics of Forest Fragments Project, Instituto Nacional de Pesquisas da Amazônial

Smithsonian Tropical Research Institute, Manaus, AM, Brazil

${ }^{2}$ Smithsonian Tropical Research Institute, Balboa, Republic of Panama

\begin{abstract}
Environmental licensing is a decision-making process that requires scientific information and has far-reaching political and economic consequences. Sound science leads to informed decisions; unfocused science leaves an information void that is easily filled by power struggles. In the last few years, Brazil has seen a lively debate between two alternative approaches to the science behind environmental licensing: one centers on detailed methodological prescription with broadly defined goals; the other builds on the precise definition of questions that focus the scientific work and allows case-by-case flexibility in methodological choices. This essay offers twelve guidelines for pursuing the second approach, formulated around the key questions of why, what, and how to sample. These guidelines illustrate how it is possible to set scientific standards of operation without tying the hands of practitioners to omnibus protocols that may serve the purpose of accumulating data but won't necessarily produce knowledge to inform rational licensing decisions. The guidelines are formulated in the context of Brazilian environmental licensing, but they should apply wherever a regulatory agency needs to elicit scientific answers to urgent environmental questions.
\end{abstract}

Key words: Environmental Licensing, Sampling, Monitoring Protocols, Management.

\section{Introduction}

In modern democratic societies, there is an expectation that large construction projects will be submitted to detailed evaluation. When the projects affect environmental resources, the evaluation is called environmental licensing. In Brazil, once a developer submits a project to evaluation, the licensing process involves three main actors: the decision-maker, who is often a political appointee in a public licensing institution; the environmental analyst, commonly an employee in the licensing institution; and the scientist, in a role usually played by a consultant that is hired more or less directly by the project developer. The decision-maker is responsible for making the final licensing decision, the analyst must provide the decision-maker with an appraisal of the project, and the scientist must answer key technical questions posed by the analyst. The answers to these questions should form the backbone of the appraisal that will support the final decision. This appraisal must contain an informative synthesis of the best available knowledge about the project's potential implications.

\footnotetext{
*Send correspondence to: Gonçalo Ferraz

Biological Dynamics of Forest Fragments Project,

Instituto Nacional de Pesquisas da Amazônia,

Av. André Araujo, 1753, Petrópolis,

CP 478, CEP 69083-000, Manaus, AM, Brasil

E-mail: ferrazg@si.edu
}

During the last five years, Brazil has seen profound changes and a lively debate over the regulation of biological sampling within the environmental licensing process. Part of the debate has surfaced in published discussions about monitoring design (Ferraz et al. 2008; Magnusson et al. 2008), but it has extended far beyond the academic realm into a series of technical workshops, courses and meetings hosted by the Ministry of Environment, the Ministry of Mines and Energy, the Ministry of Science and Technology, the World Bank, and consulting companies. As a result, the current situation is transient; so transient that no one involved can claim to do a good job by merely following the rules, because the rules are changing. Even though the licensing process culminates in a decision made by one actor - the decision-maker - the current debate centers on the interaction between the other two - the analyst and the scientist. The regulations at stake will eventually determine what questions the analyst should pose to the scientist, and what information the scientist should gather.

The Brazilian regulatory tool with most significance for the analyst-scientist interaction is the Normative Instruction or Instrução Normativa (IN) 146, published in January 2007. Despite its disproportionate focus on the rescue and destination of animals trapped in filling hydroelectric dams, 
the IN 146 was indeed conceived to regulate sampling in any sort of project with potential impacts over animal life. Only two years later, in May 2009, did the Brazilian Institute of the Environment and Renewable Natural Resources (IBAMA) amend the normative instruction to restrict its application to hydroelectric projects (Portaria Normativa 10 of May $22^{\text {nd }} 2009$ ). Until the definition of new rules, sampling in the remaining types of projects - including roads, power lines, pipelines, mining operations, ports, etc - is regulated by the resolution CONAMA \#237/94, which is less detailed than IN 146.

By confirming the almost unanimous impression that IN 146 was "inadequate for several types of licensing contexts" (my translation from the legal text) Portaria Normativa 10 stressed the need to rethink the rules. A climate of regulatory openness ensued and left licensing institutions with the responsibility of setting goals for the scientist's work on a case-by-case basis, with limited legal support. Licensing institutions thus issue non-binding guiding documents, often called termos de referência, which lay out more or less detailed guidelines for what the scientist should do in each particular project. In the federal sphere of licensing, for example, IBAMA's Licensing Directorate (DILIC) has issued termos de referencia with very detailed prescriptions of how the scientist should collect and analyze data. This emphasis on technical prescription is based on a legitimate intention to improve the scientist's work; nonetheless, it is not clear that the detailed guidelines are achieving their desired goal. The more thorough termos de referência are prescribing not just the spatial and temporal distribution of sampling points, but also the sampling techniques for each group of targeted organisms, analytical methods, and even software packages for analyzing data. Such level of detail amounts to a substantial incursion of the analyst into the scientist's work and has met active resistance from consulting scientists.

To better understand the predicament of scientists and analysts, it is important to consider the motivations behind the detailed prescriptions. The analyst's immediate goal is indeed to improve the work of the scientist, but there is also a deep-seated conviction that the accumulation of large amounts of data collected with one standard protocol in many licensing processes will eventually crystallize into information of great relevance for society. Most importantly, this belief is not rooted in the formulation of a priori questions to guide the design of standardized protocols. From the point of view of the scientist (and the consulting firm) there is often a more pragmatic approach aimed at asking whatever questions are key for a licensing decision, one project at a time. There is a conflict between the goal of standardized data accumulation for answering future questions across projects and the goal of pragmatic data collection for answering questions that may decide one licensing process. This conflict must be solved; otherwise there is a serious risk that the analyst, in a drive for enforcing data accumulation protocols, will hold back the scientist from effectively informing the licensing decision. At the same time, the analyst is responsible for enforcing a high standard of quality for the scientist's work and this quality should be protected by legal guidelines. The central problem thus, is how to elicit high-quality work from the scientist without tying his hands.

The solution must recognize that scientific answers are as good as the questions that prompt them. In the applied science of biological monitoring, as in the fundamental science of any enterprise of human knowledge, progress stems from creativity and sharp questions (Platt 1964). There is no data accumulation, no technical sophistication, and no standardized procedure that will give the analyst the answer that makes the difference if there is no fertile question at the outset. Future guidelines to replace IN 146 should aim at helping the analyst formulate an intelligent scientific mandate; i.e. they should lead to useful, technically viable termos de referência that help focus the scientific work without forcing the scientist to spend time and limited resources on standardized procedures that do not contribute effectively to the licensing decision. The new guidelines should not prescribe the means; they should clarify the ends and provide broad principles for choosing the means with flexibility and pragmatism

Guidelines for biological sampling in licensing studies should apply to a wide variety of sampling realities. For example, Brazilian licensing jargon often emphasizes the distinction between inventory (levantamento) and monitoring (monitoramento) sampling. These two types of sampling contribute to different parts of the three-stage licensing process, which starts with a pre-license (licença prévia), followed by an installation license (licença de instalação), and ends in a cycle of operation licenses (licença de operação) that must be renewed every three to five years. Inventories - in the licensing jargon - usually take place in the context of environmental impact studies. They offer a static characterization that precedes the issuing of a pre-license and characterize some broad aspect of local biological diversity at one point in time - that is, they aim for a static characterization. Monitoring, on the other hand, is purposefully geared towards a dynamic characterization of the study system and it is usually planned before the issuing of an installation license. Monitoring results should play a key role in the cyclic processing of operation licenses, leading naturally to the iteration of observation and decision-making that is at the core of adaptive management and monitoring designs (Williams et al. 2007; Lindenmayer \& Likens 2009). Some guidelines will inevitably be more relevant for the monitoring (dynamic) than for the inventory (static) approaches, particularly if they focus on assessing the consequences of a construction project. Nonetheless, they will all have a shared and broad relevance if they are focused on solving clearly defined scientific problems whose solution should guide the licensing decision. Such 
focus helps all sorts of sampling realities: broad or narrow, static or dynamic.

This document offers twelve guidelines for biological sampling grouped around three simple but fundamental questions about the sampling process: 'why' sampling, 'what' to sample, and 'how' to sample (Nichols \& Williams 2006). Because there has been so much debate regarding technical prescriptions of how to sample, the majority of guidelines address 'how' questions (guidelines 3-12): guidelines 3-6 focus on the time and space of sampling; guidelines 7-9 pertain to sampling design decisions of replication and control; and finally, guidelines 10-12 address issues of data analysis and inference of biological processes. The 'how' guidelines exemplify how it is possible to formulate broad principles that usefully direct the technical development of the study without binding the scientist to a narrow and limiting set of procedures. There is only one 'why' and one 'what' guideline but the small number of guidelines does not mean that these two questions are any less important. All decisions about how to sample are contingent on why and what one is sampling in the first place. This is no small detail.

\section{Why Sampling?}

\section{Guideline 1: Any biological sampling carried within the licensing of a construction project must stem from a preliminary assessment of the impacts of the project.}

Even when there is a broad goal of quantifying biodiversity, no one can sample every group with every possible technique. It is essential to start from some prediction of what are going to be the most important impacts of a project and such preliminary assessment must provide the foundation for deciding what and how to sample. This guideline is the most important of the list. Sometimes, information may be too scant to allow a precise prediction of what species will be affected; nonetheless, information about the construction project itself may be sufficient to anticipate broad but sufficiently informative predictions to direct the choice of focal groups, independently of prior knowledge about the local fauna. For example, roads may lead to roadkill; dams may interrupt fish migration routes; and mines may result in stream water pollution. These things are plausible even when we don't know what mammals, fish, and stream invertebrates live in the study area. Of course the road may do more than accidental killings, for instance, it may also facilitate an increase in deforestation with associated loss of habitat for all forest species. Thus, each construction project may have a considerable variety of potential impacts. Sampling must be preceded by a written preliminary identification of potential impacts and a cautious assessment of priorities among those impacts.

\section{What to Sample?}

\section{Guideline 2: The choice of sampling target must adequately reflect the preliminary impact assessment.}

With a preliminary impact assessment and a ranking of priorities in hand, the study should focus on sampling focal groups of organisms or any biological variables that best indicate the potential impacts. Merely setting out to do the best possible characterization of biodiversity in the project area is insufficient and inadequate. 'Biodiversity' is a very broad concept that embraces multiple metrics of variety, from the genetic diversity of a population to the variety of ecosystems in a geographical region and including the variety of species under the influence of a project (Hunter et al. 1998, p. 934). To characterize every aspect of biodiversity is an unattainable goal in general, much less in the short time frame of a licensing study. Scientist and analyst have the responsibility of identifying informative focal groups. It should be clear, however, that focal groups do not have to be narrow. Sampling may target one particular species, but it may also aim at a broader, even scantly known group of organisms that inhabit one particular environment. For example, if one is concerned that the slowing flow of water entering a hydroelectric reservoir may result in sediment accumulation and consequent impact on the aquatic ecosystem upstream from the damn, it seems more sensible to sample benthonic and sediment fauna rather than small arboreal rodents of the margin. Both groups are broadly defined but the first is more pertinent than the second. Finally, when sampling organisms, it is important to define not only the group but also which aspect of the group is to be observed. One may want to assess the number of species, the number of individuals of a group per unit area, or the probability that individuals from a given group are found dead, to name only a few possibilities. Note that not all of these demand a detailed knowledge of group taxonomy. The choice of metric will reflect on the study's ability to assess impacts and will have important consequences on the decision of how to sample.

\section{How to Sample? Space and Time}

\section{Guideline 3: The sampling area must represent the area under the influence of the construction project.}

Regulations for biological sampling in environmental licensing cannot set the correct sampling area or location for all studies. That is obviously not possible because the appropriateness of a sampling area will depend on the nature of the project, on the predicted impacts, and on the focal group. What the regulations can do is to require that placement and size of the sampling area be justified with a logical connection to the characteristics of the project 
surroundings, the predicted impacts, and the sampling target. For example, the terminology for environmental impact studies for hydroelectric dams includes the concepts of 'direct influence' and 'indirect influence' areas. This guideline should motivate the careful definition of different types of influence areas as well as the identification of sampling areas that adequately represent them, on a case-by-case basis. Prescribed limits should not be used for excluding important areas.

\section{Guideline 4: The grain of sampling must be compatible with the biology of the sampling target.}

The concept of grain in ecology (Wiens 1989) is analogue to the idea of resolution in a digital photograph: five megapixels means a finer grain than two megapixels. In field sampling, a fine grain amounts to shorter distances between sampling points. In practice, a fine-grain study is bound to cover a limited area, which implies a tradeoff between sampling area (guideline 3) and grain (this guideline). When sampling organisms, the choice of grain should be based on the natural history of the target group. Organisms with a small home range should be sampled at a finer grain than those with a large home range. In the absence of home range information, body size can be used as a proxy. The most important consequence of this guideline is that it does not make sense to impose a standardized sampling grid for all animal groups. While a network of fifty traps regularly distributed throughout $1 \mathrm{~km}^{2}$ could provide useful information for estimating the size of a frog population, there would be little to learn about jaguar population size from a network of 50 camera traps distributed throughout the same area. What works for the frog won't work for the jaguar and vice-versa. One should not expect narrow regulations to determine the grain of sampling across groups; a broader guideline like this one aims to guard against sampling plans with blatantly inadequate grain.

\section{Guideline 5: The duration of sampling in time} must be compatible with the life cycle of the sampling target and with the predicted temporal reach of the project's impact.

This guideline applies to pre-license surveys and to longerterm monitoring studies in different ways. In a survey aiming at the static characterization of a target group, it is important that all sampling takes place during a time sufficiently short to avoid substantial biological changes. If the time is too long it won't be fair to attempt a static characterization based on information that is inherently dynamic. When the aim is to monitor temporal changes that may have occurred in response to a project, sampling should be organized in a sequence of discrete periods. The time between periods must be long enough to accommodate the type of changes that were anticipated. The periods, in turn, should be as short as a corresponding survey period and for the same reasons (Williams et al. 2002; chapter 19). For example, suppose a project is predicted to reduce the population of a target group of animals that survive for several years and this reduction is expected to happen within two to ten years of construction. In this context, it is not necessary to sample seasonally (e.g., twice a year) and obtain estimates of population size every six months. It is more sensible to save resources and sample once a year. A yearly sampling plan could focus on the season when animals are most active and easiest to detect, yielding yearly population estimates that suffice to access the predicted effect. As with guideline 4, it becomes clear that one should not attempt to issue mandatory sampling schedules to be applied across groups with different natural histories and across projects with different predicted impacts.

\section{Guideline 6: When the study aims to access temporal changes, sampling must start before the onset of predicted impacts.}

This guideline applies to monitoring plans aimed at testing impact predictions through time. The best way to infer an impact is to compare post-construction data with pre-construction data from the same place. In the absence of pre-construction data, one can try to infer changes from the comparison of impacted-site, post-construction data with control post-construction data, i.e. data from sites supposedly not impacted by the project. This approach yields relatively weak conclusions, however, because it relies on the assumption of no-impact in control sites. The inference about temporal changes becomes stronger when there is pre- and post-construction data both from impact and from control sites, as explained in the next guideline. This topic is developed at length in the literature about Before After Control Impact (BACI) designs (Stewart-Oaten et al. 1986; Underwood 1994).

\section{How to Sample? Control and Replication}

\section{Guideline 7: When evaluating a potential impact, sampling must occur both in predictably impacted areas and in control areas, with adequately justified locations.}

The documentation of an impact is especially convincing when there is evidence of its absence in a control area that is not affected by the construction project. This condition is as important as the pre-construction sampling mentioned in guideline 6 . In practice, the definition of a control site is always a subjective matter but the decisions behind the choice of site(s) should be made as clear as possible. It is probably not a good idea to issue strict rules for the definition of a control site, but the regulations should ensure that control sites exist and their selection is carefully justified. It would be desirable to have some level of 
dialogue between scientists and analysts with regard to the definition of control sites. This dialogue would help the licensing process by circulating information between actors, potentially improving everyone's ability to interpret the scientific results. Note that, to a considerable extent, this guideline is also applicable in the context of pre-license surveys. If, for instance, one wants to show that the species richness of a focal group is as high inside as outside the area affected by a project, the final result depends on the adequate definition of what is inside and what is outside. In such definition, the 'outside' area is analogue to a control area in the monitoring context.

\section{Guideline 8: The samples must be sufficiently replicated, both in impact areas and in control areas.}

This is likely the most technically demanding guideline because it requires some abstraction and quantitative analysis prior to data collection. To implement this guideline one must consider: i) what is the desired precision of the quantitative answer that one aims to obtain; and ii) what analytical method will be employed to obtain that answer. From here, one must proceed in a manner akin to power analysis in order to find out, given one anticipated answer, what is the sample size that will achieve the desired precision. The usefulness of this exercise cannot be overrated, not only because it gives a foundation for establishing the number of sampling replicates, but also because it forces the practitioner to formally anticipate the uncertainty associated with the final result. The paper by MacKenzie \& Royle (2005) offers one example of how to compute the sample size necessary to achieve a desired precision in the context of site-occupancy estimation. Once again, it becomes clear that a useful regulation does not impose a standardized sampling design across animal groups and types of projects. As with the sampling area, the grain, and other spatial/temporal aspects of sampling design, the most adequate sample size depends on details of the study. In this case, it depends above all on the precision that we desire to achieve. The more precision one wants, the more replicates are necessary.

\section{Guideline 9: The sampling design must include some means of addressing imperfect detection or, at least, a strong argument for the infallibility of the detection process.}

Most biological sampling plans will necessarily depend on the detection of organisms in the field. As a result, the sampling results inevitably reflect the combination of two processes: a biological process that determines whether organisms are available for detection at a given site, and a sampling process that determines whether the detection of available organisms was successful or not. Many practitioners in the licensing world assume that detection is perfect, i.e. that all organisms present at a site are detected during a sample of that site. This is a flawed assumption that can be brought to question by the most rudimentary field experience: mice may not like the bait in a trap, birds may be quiet during a point count, and agouties may happen to not walk in front of a camera trap. The literature on analysis of animal population has abundant examples of how to address imperfect detection with distance data (Buckland 2001), mark-recapture data (Williams et al. 2002), presence-absence data (MacKenzie et al. 2006), or with several types of data analyzed under a Bayesian framework (Royle \& Dorazio 2008; Kéry \& Schaub 2011). Most (though not all) solutions in the literature involve repeated samples of a set of sites during a short period of time. If a scientific study based on field sampling of animal populations does not present a means of addressing detection problems, it should at least present an argument to support the notion that detection failure does not seriously affect the results.

\section{How to sample? Data Analysis and Modeling}

\section{Guideline 10: The study must include and justify the measurement of covariates that potentially interfere with inferences necessary for the licensing process.}

Imagine a projected road that will run across a sector of the mountainous Brazilian Atlantic Forest. The road's proposed trajectory took into account the needs for access to local towns as well as a variety of engineering considerations. Beyond these two concerns there is a legal obligation to conserve the population of a frog species that is only known from this particular tract of forest. The frog favors a vegetation type that occurs preferentially at high altitudes but there is considerable uncertainty about its spatial distribution. As a condition for the installation license the developer will have to set up a sampling program to monitor the occurrence of this species before and after the construction of the road. Since altitude seems to be a complicating factor, in addition to comparing pre- and post-construction data, the monitoring program should also take into account the altitude of the road. 'Before' and 'after' samples should be taken across the altitudinal range of potential frog habitat along the trajectory of the road because altitude is recognized as an important covariate of the species occurrence. Neither the analyst nor the law can prescribe the relevant covariates for all licensing process. The possibilities are so vast that it doesn't even make sense to stipulate a broad a priori set of covariates to be measured in all construction projects of a given type. Such a strategy would risk wasting limited resources in pointless data accumulation and would probably still miss relevant covariates that would be accidentally left out from the list. This guideline proposes, not imposing a list of covariates, but requiring that each study, on a case-by-case 
basis, identifies and justifies a number of covariates that are considered to be important.

\section{Guideline 11: When possible, the study should formulate and test different models representing alternative impact scenarios.}

Perhaps the most fruitful side effect of guideline 1, which recommends a preliminary impact assessment, is that it motivates dialogue between stakeholders who may have radically different opinions about the consequences of a project. The dialogue between parts holding different opinions is a key ingredient for the preliminary assessment of impacts. Such dialogue naturally leads to alternative scenarios that may substantially improve the technical content of the licensing process. For example, imagine that an appraisal of a hydroelectric project is strongly dependent on the predicted impact of the dam upon populations of migratory fish. Suppose further, that the project developer states that the most serious barrier for fish migration is the dam itself and that this problem can be avoided with a fish bypass. There is a group of conservationists, however, who argue that fish bypasses will not be enough. Their point is that fish loose their sense of orientation in slow moving water and therefore, the still waters of the reservoir will be as serious a barrier as the wall itself. Once these two alternative scenarios are identified, scientists can design a fish monitoring program to help distinguish between the two mechanisms that potentially limit fish movement: dam alone, or dam plus slow-moving water? Such design could include sampling in slow-moving waters away from dams or near fish-blocking, run-of-the-river dams with minimal water storage. This incidentally illustrates how decision-making could benefit from sampling in a variety of locations near to or far from the proposed construction site. With the two movement scenarios embodied in two alternative models, it should be easy, at the data analysis stage, to compare the relative contribution of each model to explain the data.

\section{Guideline 12: The study must include a quantification of uncertainty about its results, as well as a clear explanation of how that quantification was obtained.}

Regard uncertainty as the opposite of precision and it becomes understandable how sampling replication (guideline 8) and improved detection (guideline 9) contribute to reducing uncertainty. The quantification of uncertainty about scientific results is so important in practice that even though it was already mentioned indirectly on guidelines 8 and 9, we revisit it here with a guideline of its own. Consider the following scenario: there are two alternative routes for a natural gas pipeline and one wants to choose the route that will affect the smallest possible number of species in a focal group of vertebrates. The scientific work could start by estimating the number of species along each route. In the absence of other criteria, the analyst's recommendation could be to build the pipeline along the route with the smaller number of species. The work doesn't end here, however, because we know that uncertainty about species distribution and uncertainty about the sampling process reflect upon uncertainty about the final estimates of species numbers. This uncertainty, which can be expressed by a confidence interval around the number of species, has profound implications for decision-making. Suppose route 1 has 45 species and route 2 has 60 ; however, the cost of route 1 is 1.5 times that of route 2 . This is a situation where the numbers may lead to expensive decisions. If the species richness estimates with their associated uncertainty are, for instance, $45 \pm 5$ for route 1 and $60 \pm 7$ for route 2 , there is a strong reason to recommend the more expensive route 1 because the numbers are clearly different. If, on the other hand, the estimates were $45 \pm 14$ for route 1 and $60 \pm 12$ for route 2 , one cannot reject the possibility that the two routes have the same number of species and it becomes more difficult to argue for the most expensive option. It is absolutely key that scientific studies in the licensing process recognize and discuss the sources of uncertainty that condition the scientist's and the analyst's degree of belief in the final results. At the very least, these sources of uncertainty should be verbally acknowledged, but the results will be much more helpful if they include a quantitative measure of uncertainty. This measure should be informatively described in the scientific study.

\section{Conclusion}

The guidelines presented here illustrate the range and complexity of decisions that must be made during the biological studies that support licensing decisions. They are all relevant but the importance of guideline 1 - formulation of a preliminary impact assessment - cannot be overemphasized. Without such prediction there is no foundation upon which to implement the other guidelines and, consequently, no criteria to decide on what and how to sample.

How can one adequately assess the impacts of a project before they take place? It is certainly fundamental to have the best possible knowledge of the biological, socio-economical, and technical background of the project. To a certain extent, it is possible to risk predictions based on the literature and on experience from other projects. An accumulation of such external information may lead to draft lists of usual impacts by type of project and those lists may provide useful reference for the scientific work. Eventual lists of likely impacts, however, should be seen as reference material and not as a base for mandatory sampling of such and such organisms or biological variables in such and such way. Because each process has its own complex reality one must not try to automate the decisions of what and how to sample. Instead, it is desirable that a preliminary impact assessment stems from a dialogue between the stakeholders in the licensing process, regardless of their standing pro 
or against the project. Such dialogue should result in a clear technical understanding of why and how the project should or should not be licensed from the point of view of each stakeholder. This understanding should be structured around each stakeholder's prediction of the consequences of the project and thus contain the preliminary impact assessment that would serve as the foundation for the ensuing scientific work.

There is one closing concern: is it reasonable to expect that analysts and scientists in the pertinent licensing context have the technical skills to implement these guidelines? The answer may be 'not yet' but this should not discourage the application of the guidelines. This document discussed the partnership between analysts and scientists in the elaboration of biological studies. The same partnership could be extended from study elaboration to study evaluation. Scientists from research institutions could be asked to review licensing studies and to comment on the fulfillment of technical guidelines in a process comparable to peerreview. Many scientists who are not actively involved in licensing work already contribute their knowledge to public decision processes with different degrees of formality. The occasional evaluation of licensing studies would be one more opportunity to fulfill a social obligation and, with the right incentives, an opportunity for professional progress. Furthermore, the guidelines would provide a strong incentive for technical improvement among scientists and analysts already working in the licensing world.

\section{Acknowledgements}

This essay was stimulated by conversations with Rodrigo Koblitz and Ana Elisa Bacellar Schittini about the desirability of omnibus monitoring designs, during an occupancy estimation workshop at IBAMA's Licensing Directorate, in Brasilia. Frederica Sodré, Elisangela M. Almeida, and C. Frederico Menezes, from the Empresa de Pesquisas Energéticas provided key feedback and information about Brazilian licensing regulations. Carlos Grelle and one anonymous reviewer offered insightful revisions to the manuscript. I am thankful to all students and colleagues who have contributed to the lively ongoing debate about biological monitoring and its management implications. This is contribution 590 of the BDFFP technical series.

\section{References}

Buckland ST, 2001. Introduction to distance sampling: estimating abundance of biological populations. Oxford: Oxford University Press.
Ferraz G, Marinelli C \& Lovejoy TE, 2008. Biological monitoring in the Amazon: recent progress and future needs. Biotropica, 40:7-10. http://dx.doi.org/10.1111/j.1744-7429.2007.00355.x

Hunter D, Salzman J \& Zaelke D, 1998. International environmental law and policy. New York: Foundation Press.

Kéry M \& Schaub M, 2011. Bayesian population analysis using WinBUGS: a hierarchical perspective. Amsterdam: Academic Press.

Lindenmayer DB \& Likens GE, 2009. Adaptive monitoring: a new paradigm for long-term research and monitoring. Trends in Ecology and Evolution, 24:482-486. http://dx.doi. org/10.1016/j.tree.2009.03.005

MacKenzie DI \& Royle JA, 2005. Designing occupancy studies: general advice and allocating survey effort. Journal of Applied Ecology, 42:1105-1114. http://dx.doi. org/10.1111/j.1365-2664.2005.01098.x

MacKenzie DI et al., 2006. Occupancy Estimation and Modeling: Inferring Patterns and Dynamics of Species Occurrence. Amsterdam: Academic Press.

Magnusson WE et al., 2008. A program for monitoring biological diversity in the Amazon: an alternative perspective to threat-based monitoring. Biotropica, 40:409-411. http:// dx.doi.org/10.1111/j.1744-7429.2008.00427.x

Nichols JD \& Williams B, 2006. Monitoring for conservation. Trends in Ecology and Evolution, 21:668-673. http://dx.doi. org/10.1016/j.tree.2006.08.007

Platt JR, 1964. Strong inference - Certain systematic methods of scientific thinking may produce much more rapid progress than others. Science, 146:347-353. PMid:17739513. http:// dx.doi.org/10.1126/science.146.3642.347

Royle JA \& Dorazio RM, 2008. Hierarchical modeling and inference in ecology. Amsterdam: Academic Press.

Stewart-Oaten A, Murdoch WW \& Parker KR, 1986. Environmental Impact Assessment: "Pseudoreplication" in Time. Ecology, 67:929-940. http://dx.doi.org/10.2307/1939815

Underwood A, 1994. On beyond BACI: sampling designs that might reliably detect environmental disturbances. Ecological Applications, 4:3-15. http://dx.doi.org/10.2307/1942110

Wiens JA, 1989. Spatial scaling in ecology. Functional Ecology, 3:385-397. http://dx.doi.org/10.2307/2389612

Williams B, Nichols JD \& Conroy M, 2002. Analysis and management of animal populations. San Diego: Academic Press.

Williams BK, Szaro RC \& Shapiro CD, 2007. Adaptive management: the US Department of the Interior technical guide. Washington: US Department of Interior.

Received: December 2011

First Decision: January 2012 Accepted: January 2012 